\title{
Dendrimer Conjugation Enhances Tumor Penetration and Efficacy of Doxorubicin in Extracellular Matrix-Expressing 3D Lung Cancer Models
}

Rashed M. Almuqbil ${ }^{1,2, \#, ~ R o d r i g o ~ S . ~ H e y d e r ~}{ }^{1,2}$, Elizabeth R. Bielski ${ }^{1, \&}$, Mikhail Durymanov³, Joshua J. Reineke ${ }^{3}$, and Sandro R. P. da Rocha ${ }^{1,2 *}$

${ }^{1}$ Department of Pharmaceutics and ${ }^{2}$ Center for Pharmaceutical Engineering and Sciences School of Pharmacy, Virginia Commonwealth University, Richmond, VA 23284, United States

${ }^{3}$ Department of Pharmaceutical Sciences, College of Pharmacy and Allied Health Professions, South Dakota State University, Brookings, SD 57007, United States

* To whom correspondence should be addressed: e-mail: srdarocha@,vcu.edu

* $\mathrm{PhD}$ candidate in Pharmaceutics at VCU; on leave from Department of Pharmaceutical Sciences, College of Clinical Pharmacy, King Faisal University, Al Ahsa, Saudi Arabia \& Current position: Chemist in the Division of Therapeutic Performance (DTP)/ORS/OGD at FDA. 


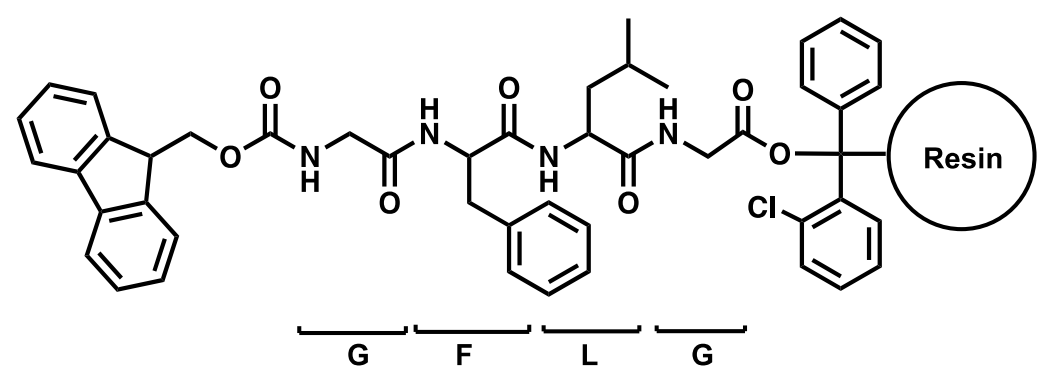

Scheme S1: Standard peptide synthesis of Fmoc-Protected peptide chain attached to 2-Cl-Trt resin (Fmoc-GFLG-Resin). G: Glycine, F: Phenylalanine, L: Leucine. 


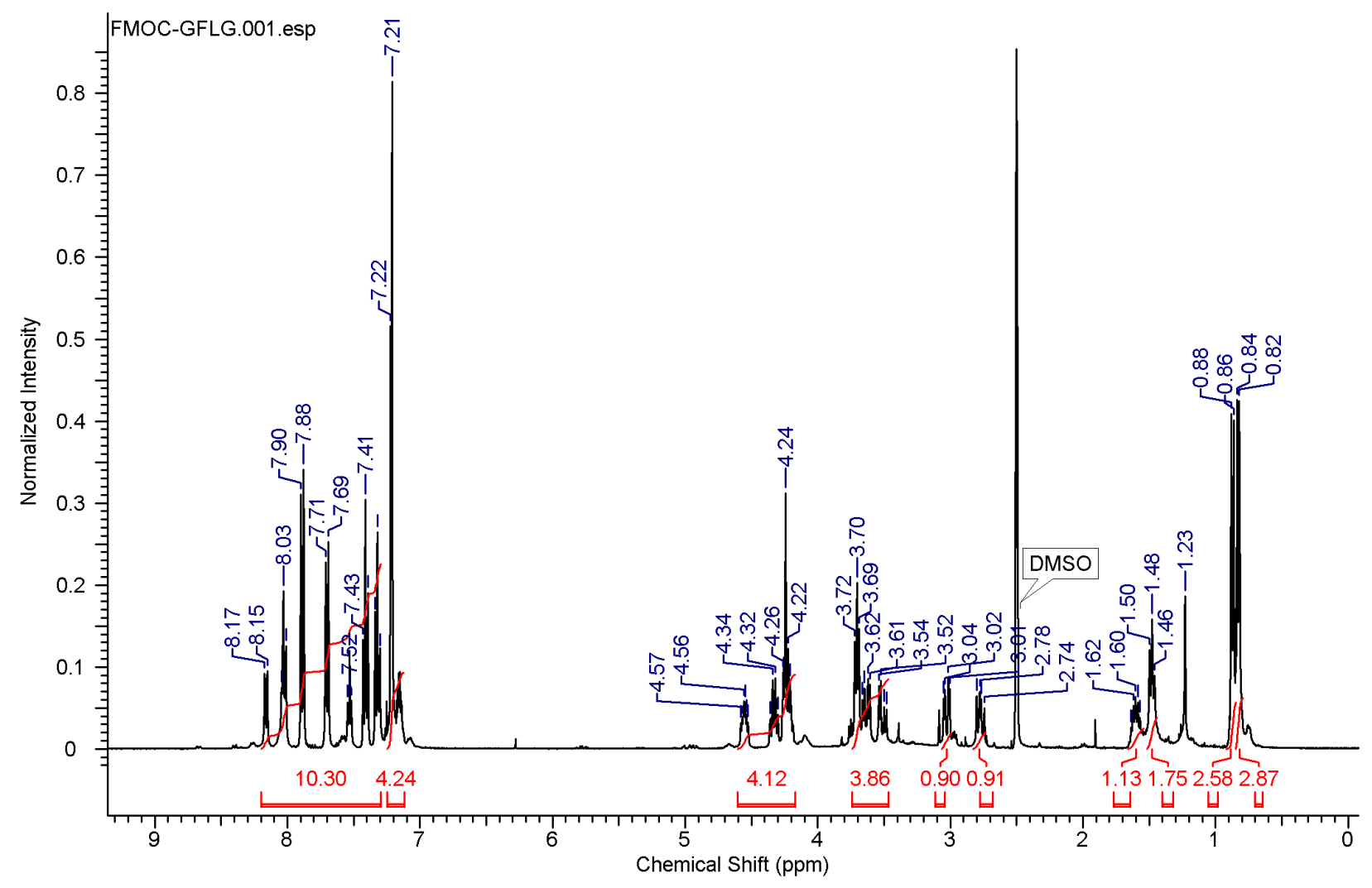

Figure S1: ${ }^{1} \mathrm{H}$ NMR $\left(400 \mathrm{MHz}, \mathrm{DMSO}-d_{6}\right)$ spectrum of Fmoc-GLFG peptide $\delta(\mathrm{ppm}): 0.83(\mathrm{~d},-$ $\left.\mathrm{CH}_{3}, 3 \mathrm{H}, \mathrm{Leu}\right), 0.87$ (d, $\left.-\mathrm{CH}_{3}, 3 \mathrm{H}, \mathrm{Leu}\right), 1.48$ (t, $\left.-\mathrm{CH}_{2}, 2 \mathrm{H}, \mathrm{Leu}\right), 1.61$ (sept, -CH, 1H, Leu), 2.78 (m, -CH, 1H, FMOC), 3.04 (dd, -CH, 1H, Leu), 3.49-3.73 (m, 4H, Gly), 4.22-4.57 (m, 4H), 7.21 (d, -CH, 4H, Phe), 7.31-8.17 (m, 9H, -CH, FMOC). 


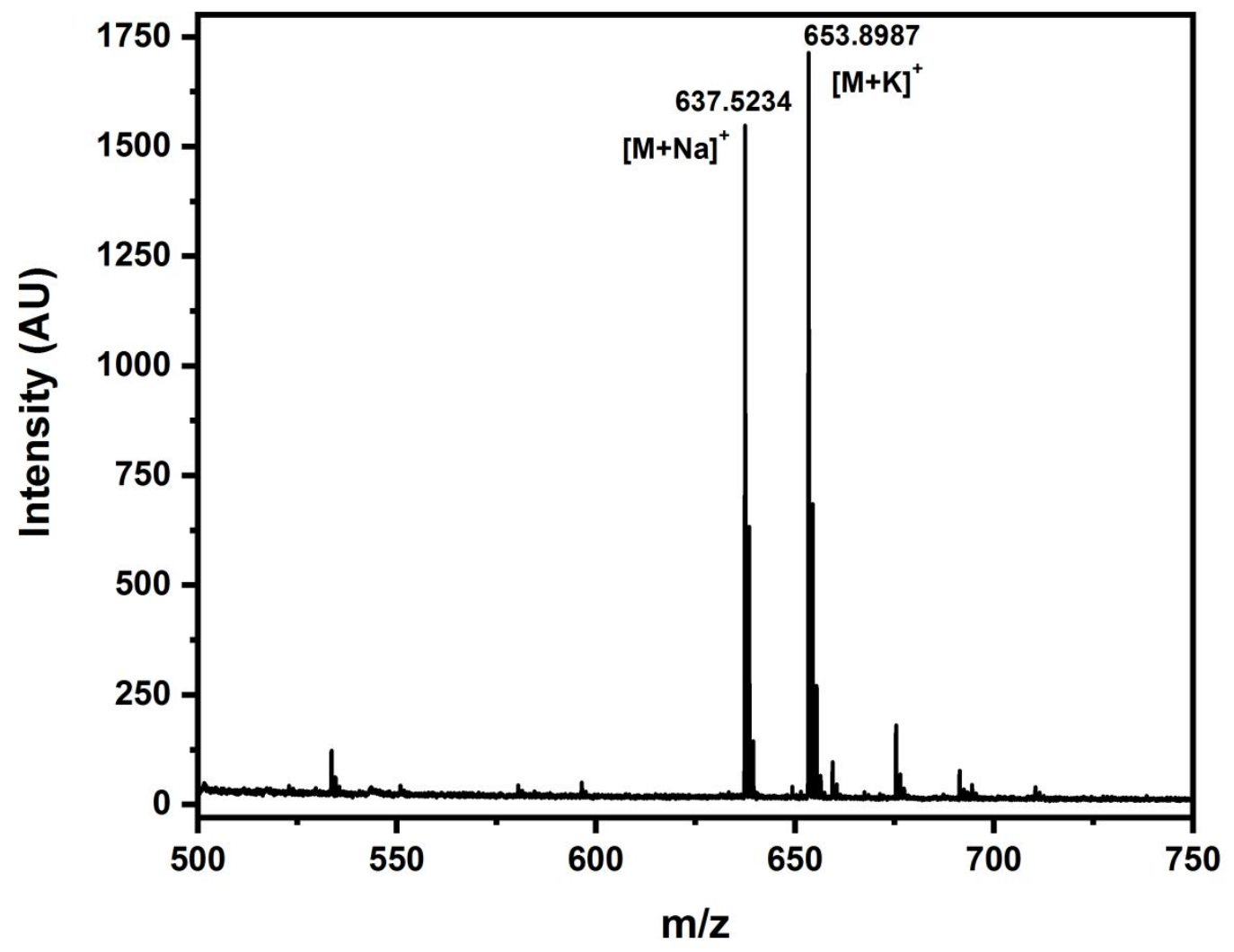

Figure S2: MALDI-ToF spectrum of Fmoc-GFLG peptide: $[\mathrm{M}+\mathrm{Na}]^{+} 637.52 ;[\mathrm{M}+\mathrm{K}]^{+} 653.90$ Calculated: 614.70 Da. 

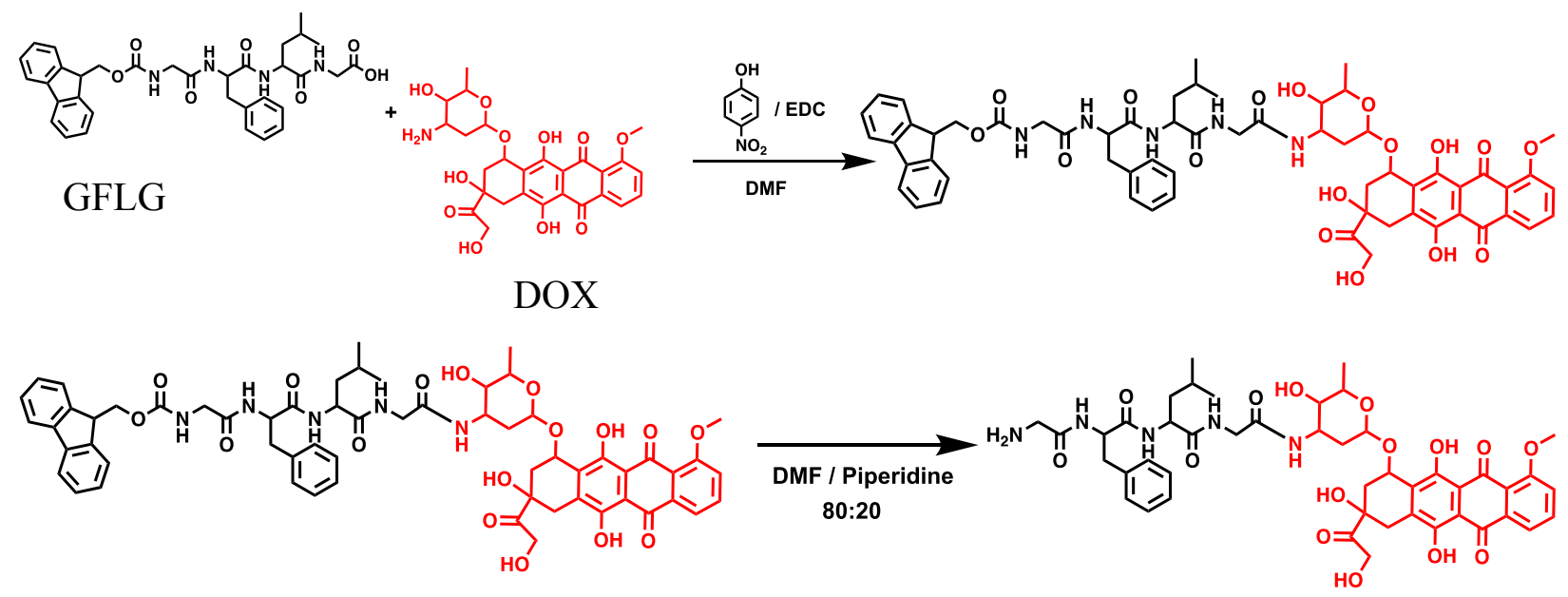

Scheme S2: Schematic of the conjugation of the GFLG peptide with doxorubicin (GFLG-DOX). EDC: ethyl-3-(3-(dimethylamino)propyl) carbodiimide, DMF: Dimethylformamide. 


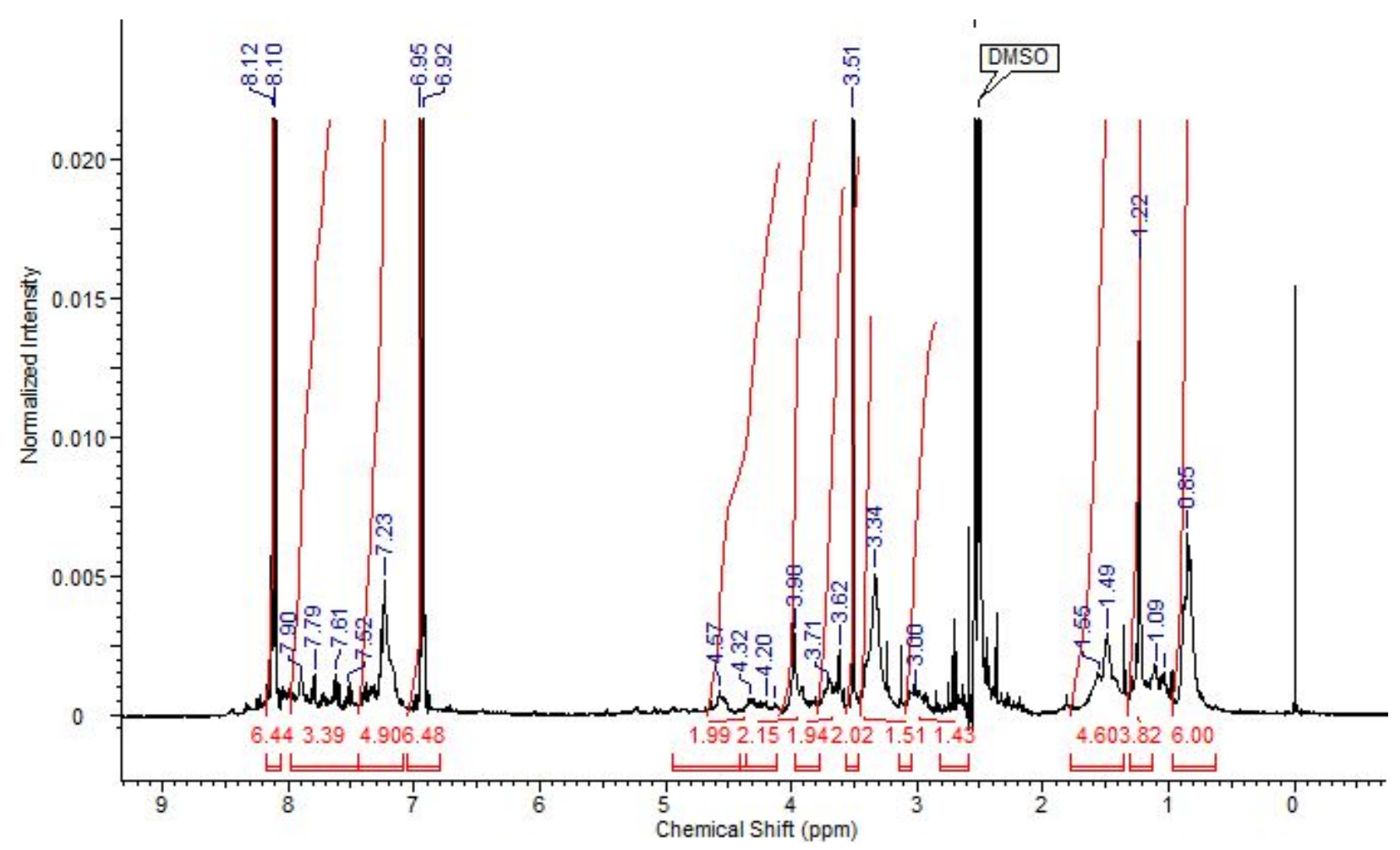

Figure S3: ${ }^{1} \mathrm{H}$ NMR $\left(400 \mathrm{MHz}, \mathrm{DMSO}-d_{6}\right)$ spectrum of Fmoc-GLFG-DOX $\delta(\mathrm{ppm})$ : 0.85 (bs, $\left.\mathrm{CH}_{3}, 6 \mathrm{H}, \mathrm{Leu}\right), 1.22$ (s, - $\left.\mathrm{CH}_{3}, 3 \mathrm{H}, \mathrm{DOX}\right)$ 1.49-1.55 (m, $-\mathrm{CH}_{2}, 2 \mathrm{H}$, Leu + sept, -CH, 1H, Leu + $\mathrm{CH}, 1 \mathrm{H}+-\mathrm{CH}, 1 \mathrm{H}, \mathrm{DOX}), 3.00(\mathrm{~m},-\mathrm{CH}, 1 \mathrm{H}, \mathrm{Leu}+-\mathrm{CH}, 1 \mathrm{H}, \mathrm{DOX}), 3.51$ (d, $\left.-\mathrm{CH}_{2}, 2 \mathrm{H}, \mathrm{Phe}\right)$, 3.62 (m, $\left.-\mathrm{CH}_{2}, 2 \mathrm{H}, \mathrm{Gly}\right), 3.98$ (s, $\left.-\mathrm{CH}_{3}, 3 \mathrm{H}, \mathrm{DOX}\right), 4.20-4.57$ (m, $-\mathrm{CH}_{2}, 2 \mathrm{H}$, Phe + - $\mathrm{CH}_{2}, 2 \mathrm{H}$, Gly + -CH, 1H, DOX), 6.94 (d, -CH, 4H, FMOC), 7.23 (m, -CH, 4H, Phe), 7.52-7.90 (m, -CH, 3H, DOX), 8.11 (d, -CH, 4H, FMOC). 


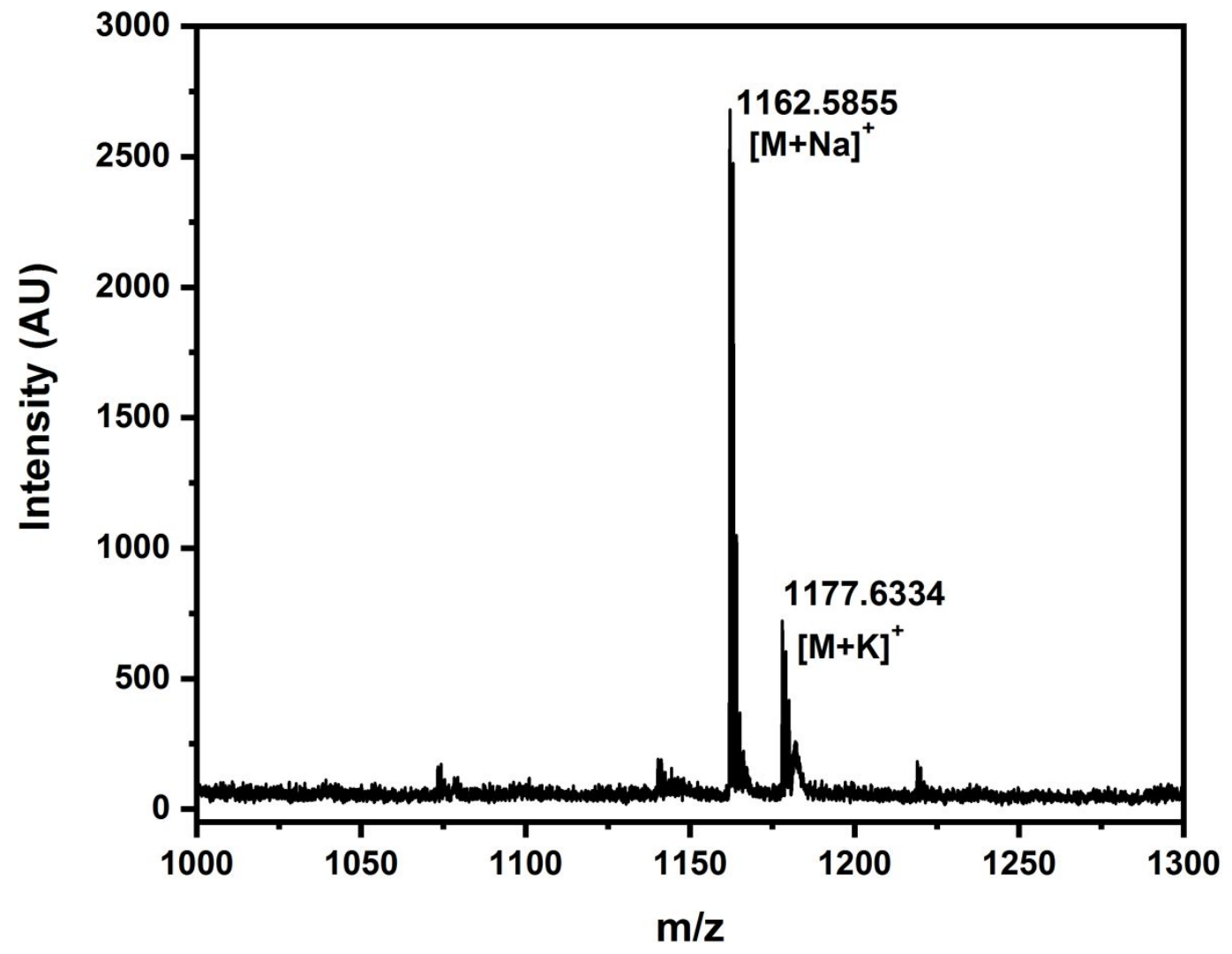

Figure S4: MALDI-ToF spectrum of Fmoc-GFLG-DOX peptide: $[\mathrm{M}+\mathrm{Na}]^{+} 1162.59$; $[\mathrm{M}+\mathrm{K}]^{+}$ 11774.63. Calculated: 1139.44 Da. 


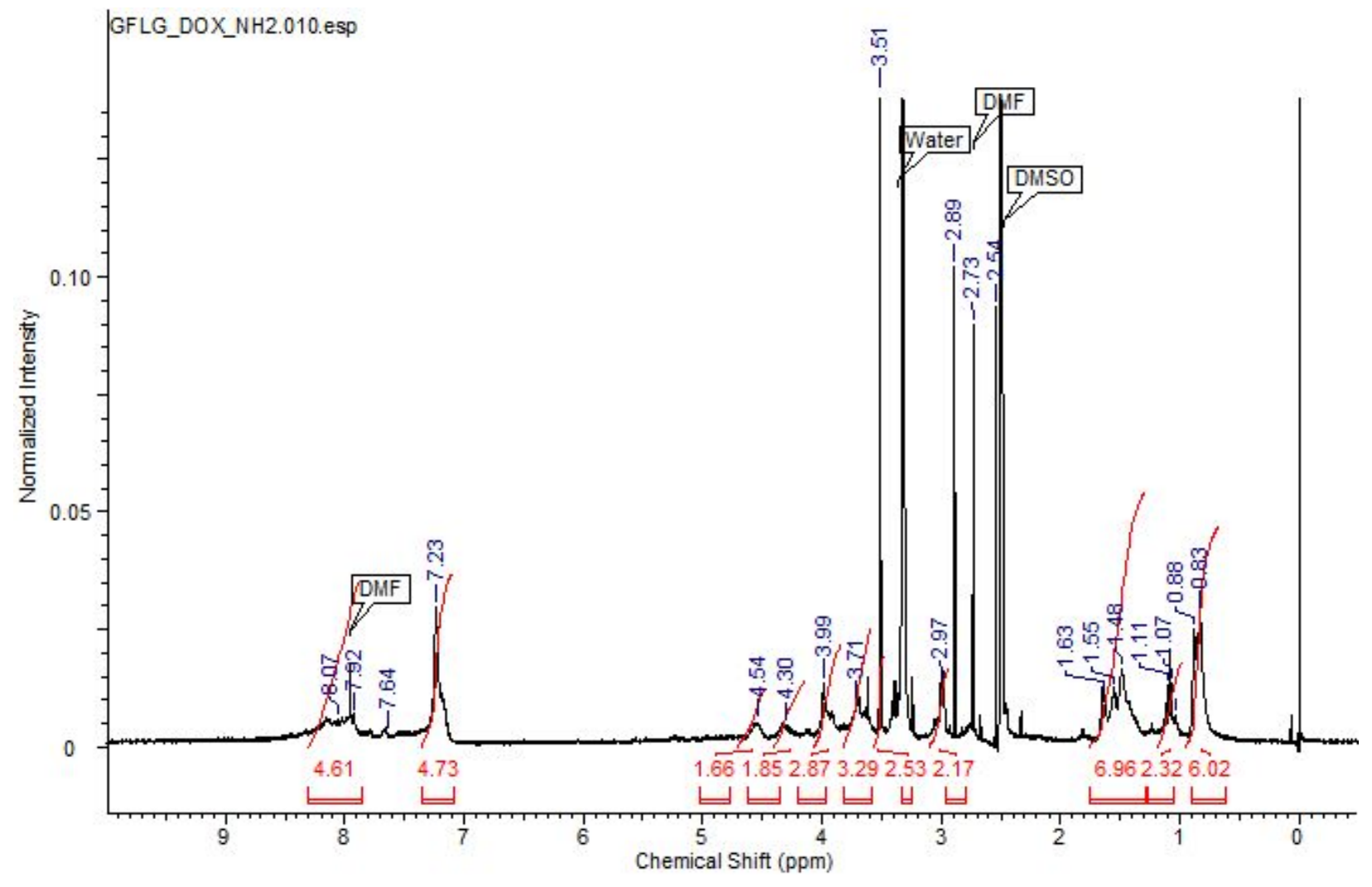

Figure S5: ${ }^{1} \mathrm{H}$ NMR (400 MHz, DMSO- $\left.d_{6}\right)$ spectrum of GLFG-DOX $\delta(\mathrm{ppm}): 0.83\left(\mathrm{~d},-\mathrm{CH}_{3}\right.$, $3 \mathrm{H}, \mathrm{Leu}), 0.88$ (d, $\left.-\mathrm{CH}_{3}, 3 \mathrm{H}, \mathrm{Leu}\right), 1.07$ (d, $\left.-\mathrm{CH}_{3}, 3 \mathrm{H}, \mathrm{DOX}\right)$ 1.48-1.63 (m, $-\mathrm{CH}_{2}, 2 \mathrm{H}$, Leu + sept, $-\mathrm{CH}, 1 \mathrm{H}, \mathrm{Leu}+-\mathrm{CH}, 1 \mathrm{H}+-\mathrm{CH}, 1 \mathrm{H}, \mathrm{DOX}), 2.97\left(\mathrm{~m},-\mathrm{CH}, 1 \mathrm{H}, \mathrm{Leu}+-\mathrm{CH}_{2}, 2 \mathrm{H}, \mathrm{DOX}+-\mathrm{CH}\right.$, 1H, DOX), 3.51 (d, - $\left.\mathrm{CH}_{2}, 2 \mathrm{H}, \mathrm{Phe}\right), 3.71$ (m, - $\left.\mathrm{CH}_{2}, 4 \mathrm{H}, \mathrm{Gly}\right), 3.99$ (s, - $\left.\mathrm{CH}_{3}, 3 \mathrm{H}, \mathrm{DOX}\right), 4.30$ (s, $\mathrm{CH}_{2}, 2 \mathrm{H}$, Phe), 4.54 (m, -CH, 1H, DOX), 7.23 (m, -CH, 4H, Phe), 7.64-8.07 (m, -CH, 3H, DOX). 


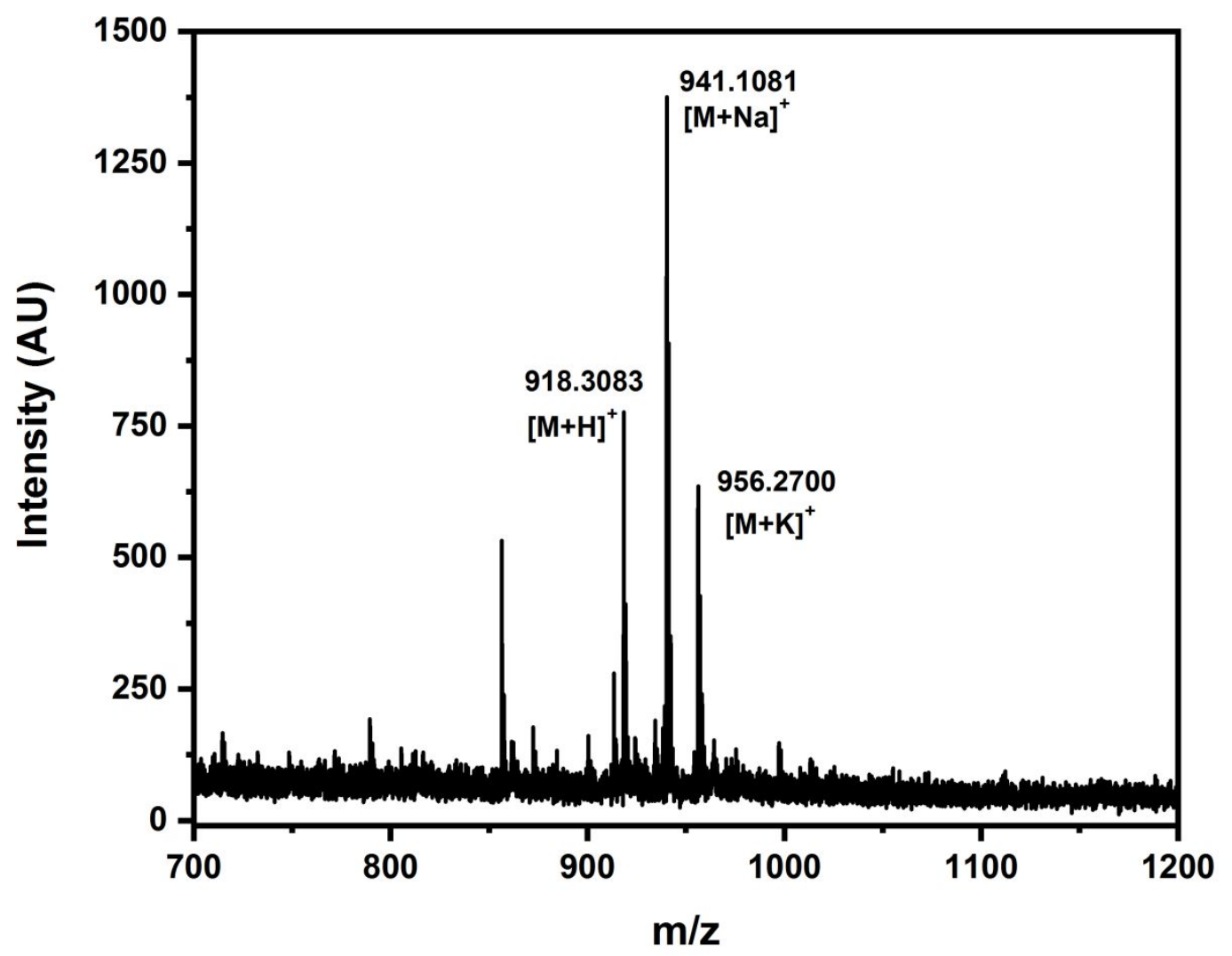

Figure S6: MALDI-ToF spectrum of GFLG-DOX: $[\mathrm{M}+\mathrm{H}]^{+}$918.31; $[\mathrm{M}+\mathrm{Na}]^{+}$941.11; $[\mathrm{M}+\mathrm{K}]^{+}$ 956.27 Calculated: 917.37 Da. 


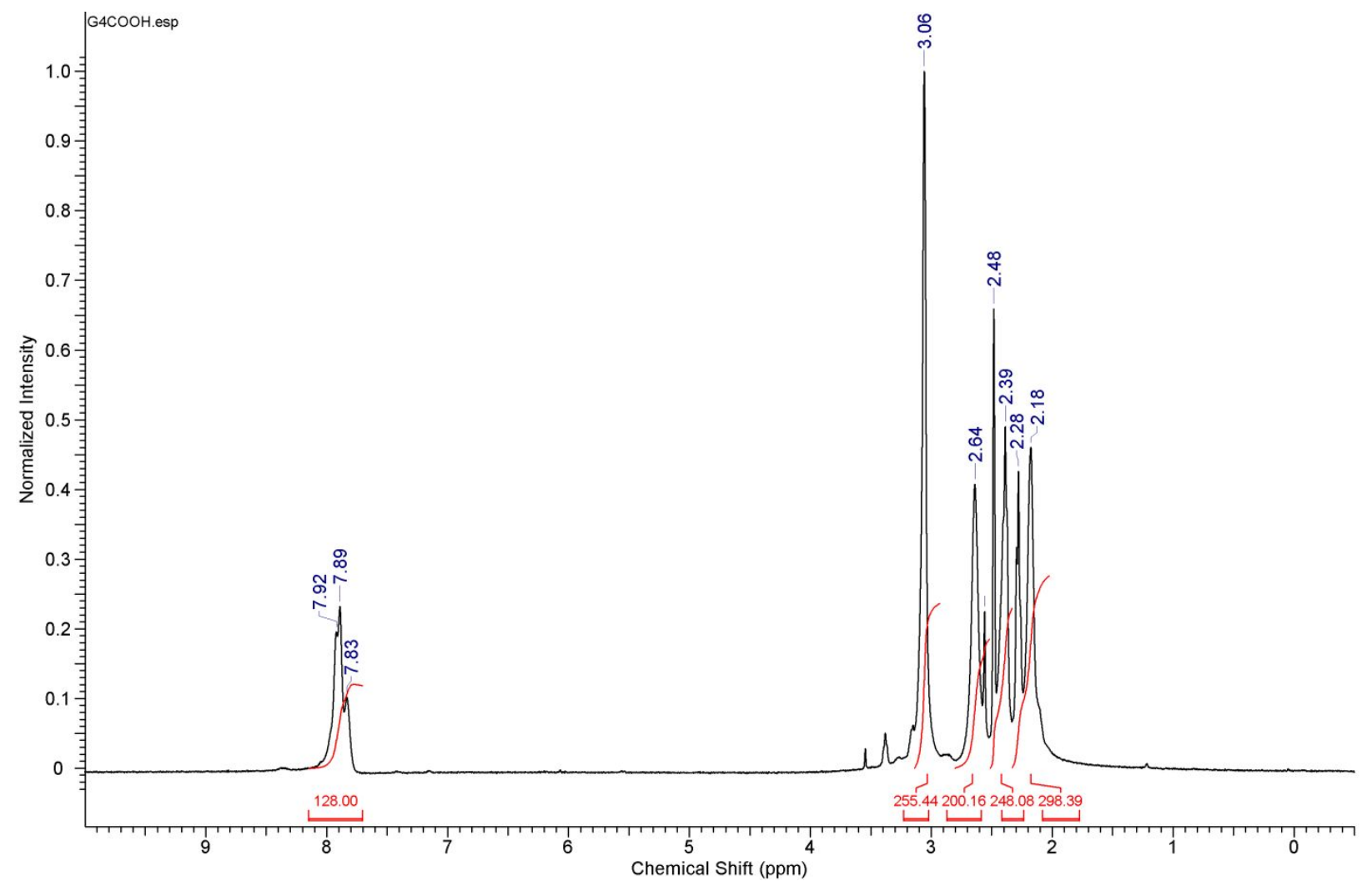

Figure S7: ${ }^{1} \mathrm{H}$ NMR spectrum (400 MHz, DMSO- $\left.d_{6}\right)$ of G4SA $\delta(\mathrm{ppm}): 2.18-2.28\left(\mathrm{~m},-\mathrm{CH}_{2}\right.$, $298 \mathrm{H}), 2.39-2.48$ (m, $\left.-\mathrm{CH}_{2}, 248 \mathrm{H}\right), 2.64$ (m, $\left.-\mathrm{CH}_{2}, 200 \mathrm{H}\right), 3.06$ (bs, $\left.-\mathrm{CH}_{2}, 255 \mathrm{H}\right), 7.83-7.92$ (m, $128 \mathrm{H})$. 


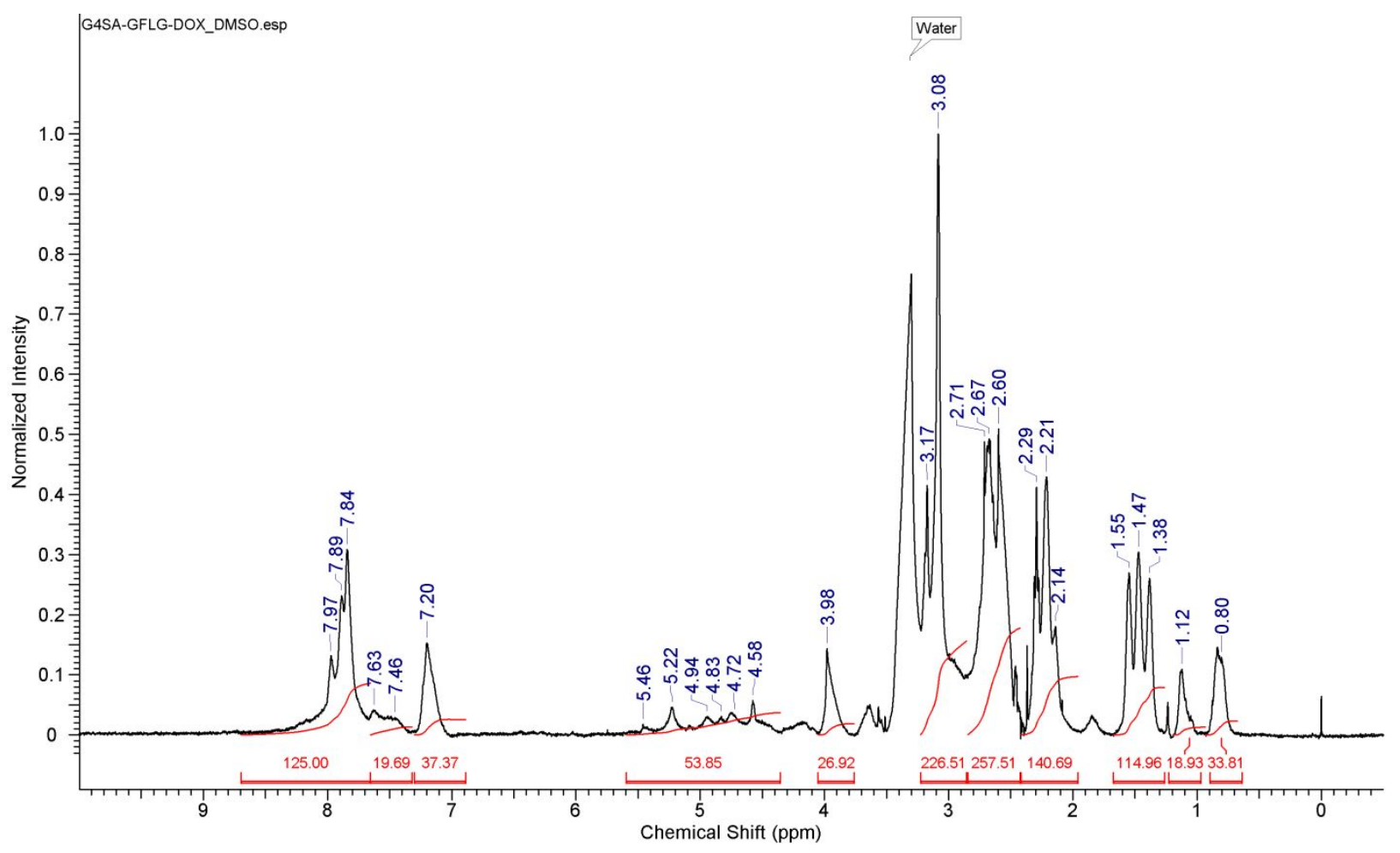

Figure S8: ${ }^{1} \mathrm{H}$ NMR spectrum $\left(400 \mathrm{MHz}, \mathrm{DMSO}-d_{6}\right)$ of G4SA-GLFG-DOX $\delta$ (ppm): 0.80 (bs, $\left.\mathrm{CH}_{3}, 34 \mathrm{H}\right), 1.12\left(\mathrm{~s},-\mathrm{CH}_{3}, 18 \mathrm{H}\right), 1.47$ (m, 140H), 2.60-2.71 (m, 257H), $3.08\left(\mathrm{~s},-\mathrm{CH}_{2}, 226 \mathrm{H}\right)$, 3.98 (s, 26H), 4.58-5.46 (m, 53H), 7.20 (bs, -CH, 37H), 7.46-7.63 (m, 19H), 7.84-7.97 (m, -NH, $125 \mathrm{H})$. 
Table S1: Number, size, and characteristics of one-week old co-culture spheroids formed by seeding different ratios and yet constant total number of cells (A549:3T3) of $220(n=4)$.

\begin{tabular}{|c|c|c|c|c|c|}
\hline \multicolumn{2}{|c|}{$\begin{array}{c}\text { Number of seeded cells } \\
\text { (day zero) }\end{array}$} & $\begin{array}{c}\text { Number of } \\
\text { Spheroids } \\
\text { (day seven) }\end{array}$ & $\begin{array}{c}\text { Surface } \\
\text { Area } \\
\left(\mu \mathbf{m}^{2}\right)\end{array}$ & $\begin{array}{c}\text { Feret's } \\
\text { Diameter } \\
(\boldsymbol{\mu m})\end{array}$ & $\begin{array}{c}\text { Other } \\
\text { Characteristics }\end{array}$ \\
\cline { 1 - 2 } $\mathbf{A 5 4 9}$ & $\mathbf{3 T 3}$ & 1 & 439.71 & 194.01 & Tight $^{*}$ \\
\hline 220 & 0 & 1 & 490.91 & 224.47 & Tight $^{*}$ \\
\hline 170 & 50 & 1 & 371.30 & 173.61 & Tight $^{*}$ \\
\hline 110 & 110 & $\&>1$ & 342.70 & 174.49 & Not as tight \\
\hline 50 & 170 & \multicolumn{3}{|c|}{ Multiple spheroids } & "Loose \\
\hline 20 & 200 & \multicolumn{3}{|c|}{ Multiple spheroids } & ${ }^{\text {\#Loose }}$ \\
\hline
\end{tabular}

* Tight spheroids $=$ spheroids that can be manipulated without being easily disrupted; "Loose spheroids = spheroids that cannot be manipulated without being disrupted. \& typically, 1 large and 1 small spheroid present in the system. 


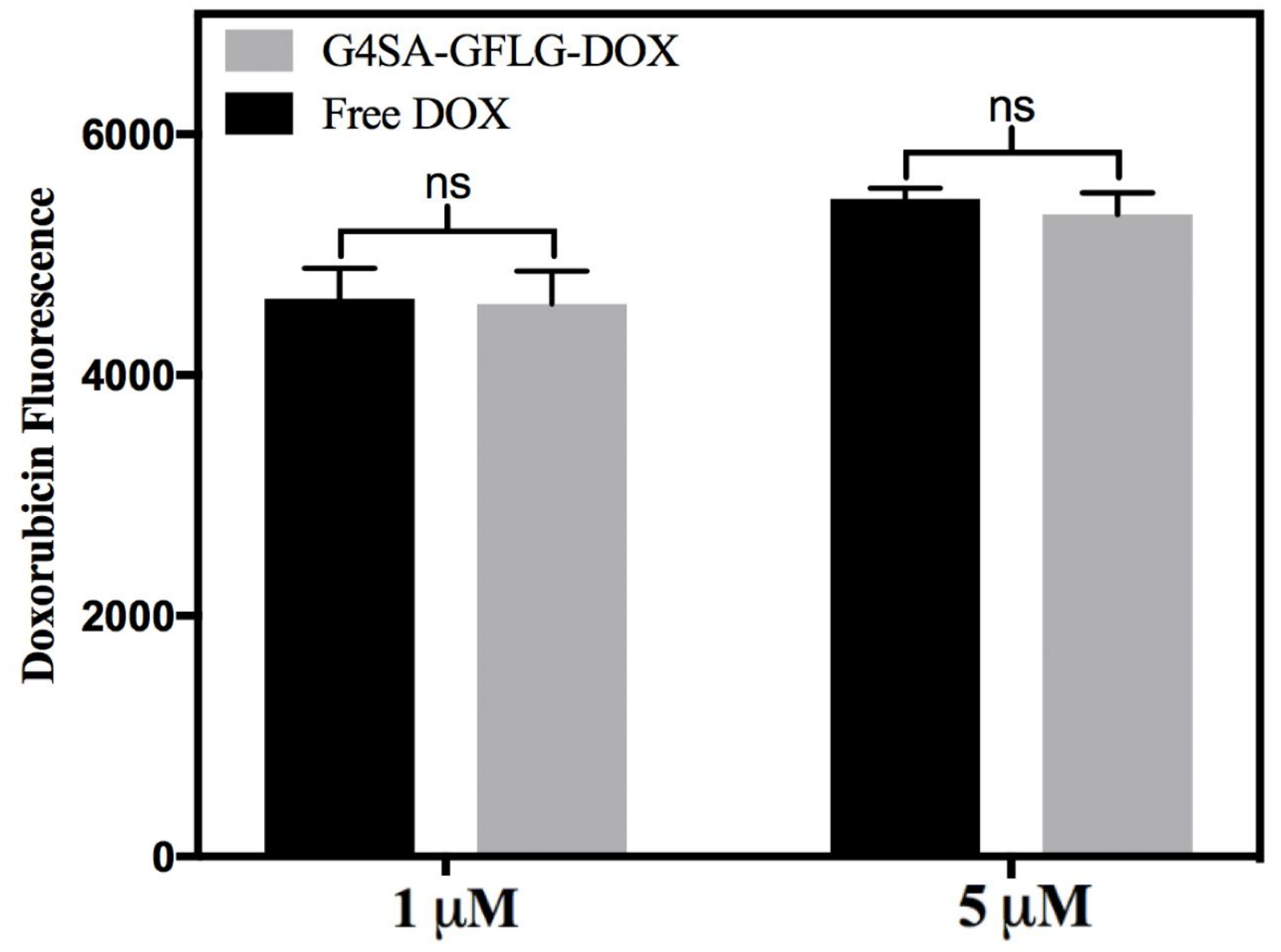

Figure S9: Fluorescence Intensity obtained from free DOX and G4SA-GFLG-DOX in HBSS. Concentration of DOX in the conjugate based on ${ }^{1} \mathrm{H}$ NMR results. No difference in fluorescence of free or conjugated DOX at maximum excitation/emission of $(470 / 590 \mathrm{~nm})$ of free DOX indicating no impact upon conjugation to dendrimer. 